\title{
The critical model size for simulating the structure- dynamics correlation in bulk metallic glasses
}

\author{
Shengjun Sun and Pengfei Guan*
}

\begin{abstract}
Computational simulation provides an effective way of understanding the disordered structure and structureproperty relationships for metallic glass systems. Here, we systematically investigated the finite-size effects of the static structure and dynamical behaviors in a three-dimensional $\mathrm{Cu}_{50} \mathrm{Zr}_{50}$ model metallic glass via classical molecular dynamics (MD) simulations. It was found that the local structure is insensitive to the system size while the dynamical properties present evident finite-size effects. The decoupling between local structure and relaxation dynamics in the investigated supercooling emerges when the system contains less than $\sim 2000$ atoms. However, the collapse can be observed between the structural relaxation time and the dynamical heterogeneity for different sized systems across the whole range of our investigation. Our results support the intrinsic link between the structural relaxation time and dynamic heterogeneity and reveal the critical simulated system size for representing the structural origins of dynamics in bulk metallic glass with ignorable surface effects.
\end{abstract}

Keywords: metallic glass, amorphous structure, structural relaxation, computational simulation

\section{INTRODUCTION}

Metallic glasses (MGs), which display unique physical properties of high strength, corrosion resistance, and excellent soft magnetic properties, have attracted extensive attention and significant research enthusiasm. Over recent decades, incessant efforts [1-4] have been devoted to unraveling the atomic structure model of MGs and the related structure-property relations for understanding the underlying mechanisms of their extraordinary properties. However, due to the limitation of spatial and temporal resolution of current experimental technologies, the absence of long-range order in the disordered structure of MGs poses a great challenge to the experimental study of atomic-level behaviors of MGs. Computational simulation provides an effective way of understanding the disordered structure and its response at the atomic level.

In the past decades [5], atomistic numerical simulations, serving as the particularly powerful tools, have revealed not only the atomic-scale subtle details of the disordered structure but also the intrinsic mechanisms of the related physical properties in MGs. By performing classical molecular dynamics (MD) simulations based on the established semi-empirical many-body potentials, such as embedded-atom method (EAM) potential [6,7], atomic structure and the related responses of the typical binary MGs, such as CuZr system, have been investigated systemically in Refs [8-12]. However, for the multicomponent systems, the precision of the semi-empirical many-body potential challenges the application of classical MD simulations. $A b$ initio molecular dynamics (AIMD) simulations based on the density functional theory $[13,14]$ provide precise atomic interactions by quantum mechanical approximation $[1,6]$ in favor of clearly delineating the local structural ordering $[13,15,16]$ of multi-component MG systems. However, the accuracy of AIMD simulations is at the expense of the attainable simulation time scale and the achievable system sizes $[17,18]$. In principle, the number of atoms in a supercell with periodic boundary conditions for AIMD simulations is restricted to 200-300 up to the limited. Take into consideration the absence of long-range order in glassy materials, the limited size of the simulated supercell may not be effective to represent the behaviors of the corresponding bulk counterparts due to the boundary effects.

In general, for computational simulations conducted with periodic boundary conditions, the system sizes are supposed to be large enough to represent the properties of the corresponding infinite system in the thermodynamic 
limit. Otherwise, certain properties will show a significant deviation from the bulk counterparts, namely, finite-size effects [19] occur. It is particularly concerning that the intrinsic length scales of static structure and dynamic heterogeneity in the liquid are developed with the increase of supercooling. Thus, the finite-size effects should be scrutinized to ensure the validity of the simulations for amorphous materials.

There have been numerous studies [20-24] that have shown the influence of finite-size effects in the supercooled states of simple liquid models, such as the Lennard-Jones system. It indicates that although the static structure scarcely shows a system size-dependence, the dynamical behaviors, such as the atomic mobility, the transient hydrodynamics, and structural relaxation, in a finite-size system manifest apparent deviation from those of larger systems. For MGs, the previous simulation studies $[25,26]$ concentrated on the size effect in lowdimensional metallic liquids and glasses. It was found that the loosely packed, liquid-like surface layers with faster dynamics play a dominant role in the strong size dependence of the atomic structure, dynamics, and glass transition temperature in low-dimensional MGs. It is of interest to investigate the size effect in a three-dimensional system with the periodic boundary condition representing the bulk MG with ignorable surface effects. Amokrane et al. [27] indicated that the system size has only a negligible effect on the atomic structure and the metallic bond characteristics in the bulk $\mathrm{Cu}-\mathrm{Ti}-\mathrm{Zr}$ system. A recent study [28] reported that the structural relaxation behaviors show an evident system size-dependence in MG-forming liquids. It implies that the simulated system size may present different influences of atomic structure and relaxation dynamics in the atomistic numerical studies of MGs. Thus, it is important to explore the system size influences of the atomic structure and various physical properties for investigating the effective structureproperty relations in MGs.

So far, there is still no systematic study on the system size effects in the simulated MGs. In this work, we investigated the system size dependences of static structure and dynamical properties for the typical binary $\mathrm{Cu}_{50} \mathrm{Zr}_{50}$ MG-forming liquid by classical MD simulations. It is proved that the structure relaxation dynamics are sensitive to the system size. The finite-size effects in the dynamics of MG-forming liquids get more pronounced with decreasing temperature. Meanwhile, the correlation between the structural relaxation time and the dynamic heterogeneity collapses on a master curve for systems with different sizes. Since the short-range order of atomic structure is insensitive to the system size, the collapse of the structure-dynamics relation in the investigated supercooling can only be obtained for systems with sizes lager than $\sim 2000$ atoms. It suggests that the simulated system size for representing the structural origins of dynamics in bulk MGs should consist of thousands of atoms, which is far beyond the limited size of AIMD simulations.

\section{COMPUTATIONAL SECTION}

We performed the MD simulations in a binary model $\mathrm{Cu}_{50} \mathrm{Zr}_{50}$ MG using the open-source code large-scale atomic/molecular massively parallel simulator (LAMMPS) [29]. The EAM potential developed by Mendelev et al. [7] was employed to describe the interatomic interactions in the study.

In our simulations, periodic boundary conditions were imposed in three dimensions of the cubic box. To evaluate the finite-size effects, we carried out a wide range of simulations in different sized systems. These systems containing 100, 250,400,800, 2000, 3200, and 6400 atoms, are denoted as N100, N250, N400, N800, N2000, N3200, and N6400, respectively.

For each system, the initial configuration was first equilibrated at $1500 \mathrm{~K}$ for at least $1 \mathrm{~ns}$ and then it was quenched by a stepwise approach to $300 \mathrm{~K}$ under the isothermal-isobaric (NPT, constant number, constant pressure, and constant temperature) ensemble, with a temperature interval of $50 \mathrm{~K}$. The effective cooling rate was $10^{11} \mathrm{~K} \mathrm{~s}^{-1}$ and the time step was $2 \mathrm{fs}$. The temperature was regulated by the Nose-Hoover thermostat [30]. To obtain the structural and dynamical properties at temperatures of interest, the samples got further relaxation at each temperature under NPT ensemble. The ensemble was altered into NVT ensemble to generate the trajectory snapshots for computing relevant ensemble-averaged quantities after the system reached a full equilibrium state. Finally, $10^{3}$ and $10^{4}$ atomic configurations were collected for further structure and dynamics analysis, respectively.

\section{RESULTS AND DISCUSSION}

\section{Static atomic structure characterizations}

We primarily studied the static structure of the MG samples with different system sizes under various temperatures. The structure was characterized by the radial distribution function (RDF), Voronoi polyhedra (VP), and the averaged five-fold local symmetry parameter $W$. The temperature-dependent system-size influences of 
static structure could be systematically quantified through these parameters.

We first calculated the radial distribution function (RDF), which directly provides an averaged description of the static structure, to check the amorphous nature of all samples. The radial distribution function is the ratio of the local number density with the overall particle number density $\rho$ at the certain radial distance $r$ from a reference particle in a homogenous and isotropic system, which is defined [31,32] as $g(r)=4 \pi r^{2} g(\boldsymbol{r})$.

The pair distribution function $g(\boldsymbol{r})$ can be computed as

$g(\boldsymbol{r})=\frac{1}{4 \pi r^{2} \rho N} \sum_{i=1}^{N} \sum_{j=1, j \neq i}^{N} \delta\left(r-\left|\boldsymbol{r}_{i j}\right|\right)$,

where $\left|\boldsymbol{r}_{i j}\right|$ is the interatomic distance between one particle and another in an $\mathrm{N}$-particle system.

Fig. 1a-c provide a direct comparison of the overall $g(\boldsymbol{r})$ of seven $\mathrm{Cu}_{50} \mathrm{Zr}_{50}$ simulated systems with various system sizes at $T=1500 \mathrm{~K}$ (representing a high-temperature liquid state), $800 \mathrm{~K}$ (representing a supercooled state) and $300 \mathrm{~K}$ (representing the glassy state), respectively. The
RDF spectra show the typical pattern of broad peaks and valleys at small distance $r$. The first peak gets sharper and higher with the degree of quenching rising. The second peak splits into two subpeaks at $300 \mathrm{~K}$, which is a characteristic of MGs [33]. The distinctions among different RDF spectra are so subtle that it can hardly be perceived.

To catch the nuance, we selected the RDFs of the largest system (N6400) as the reference data and defined the difference of the RDFs as $\Delta g(r, N)=g(r, N)-g(r, N=$ 6400). The calculated $\Delta g(r, N)$ for each sample at selected temperatures are shown in the inset of Fig. 1a-c. Almost all the $\Delta g(r, N)$ curves fluctuate around zero with a very small amplitude (less than 0.15), which implies the RDF is insensitive to the change of system size, the large discrepancy in the second peak range of $\Delta g(r, N=100)$ at $300 \mathrm{~K}$ implies a developed medium-range order in N100 sample. It was further confirmed by the snapshot of the related atomic configuration as shown in Fig. 1d, which presents partial crystallization with $B 2 \mathrm{CuZr}$ phase. It suggests that the 100 -atom system under periodic boundary condition, which was widely employed in
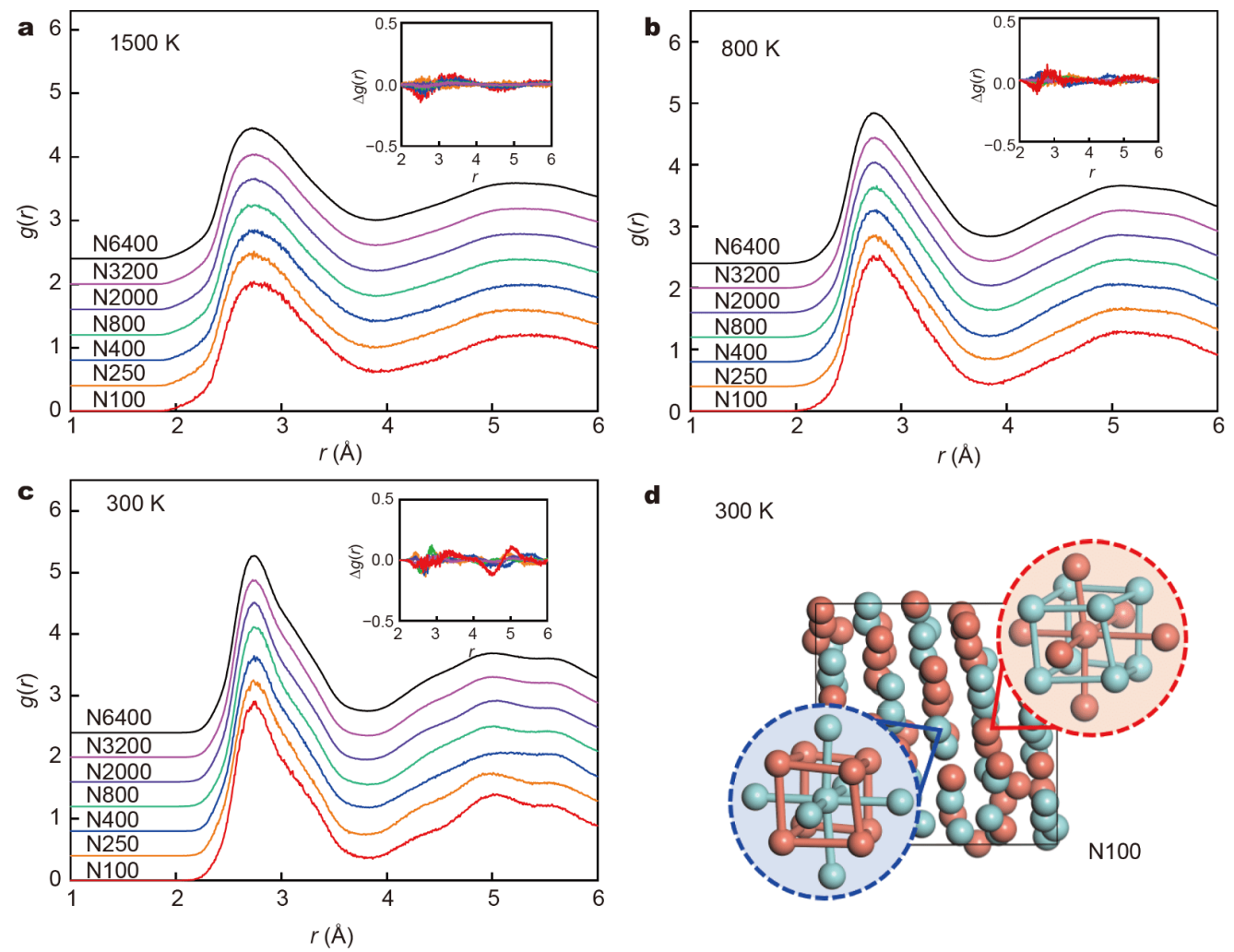

$300 \mathrm{~K}$

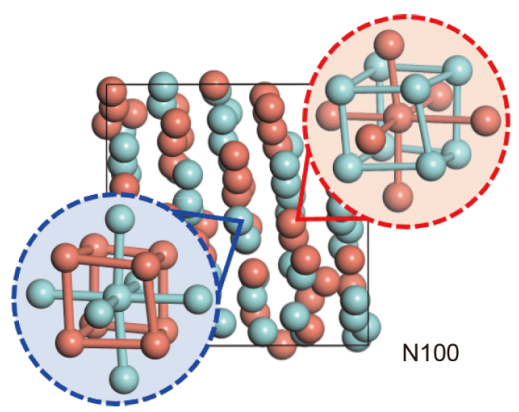

Figure 1 The simulated total radial distribution functions $g(r)$ (vertically shifted for visualization) of $\mathrm{Cu}_{50} \mathrm{Zr}_{50}$ samples with different system size $N$. $(\mathrm{a}-\mathrm{c})$ The selected temperatures are 1500,800 , and $300 \mathrm{~K}$, respectively. The RDF spectra of a given size sample were colored by the same color. The $\Delta g(r)$ of smaller samples referring to the N6400 sample are shown in the inset. (d) The snapshot of the atomic configuration with crystal-like behavior of the system containing 100 atoms (N100) at $300 \mathrm{~K}$. 
previous AIMD simulations, behaves much poor glassforming ability. It may be due to the comparable size between the simulated cell and the critical nucleation size of $\mathrm{Cu}_{50} \mathrm{Zr}_{50}$ liquid.

As we know, the radial distribution function is the onedimensional statistical average of atomic packing information that fails to render the local geometrical motifs of MGs. Recently, the Voronoi tessellation method [34] was employed to distinguish the types of local geometrical motifs in previous simulation results $[35,36]$.

Based on the Voronoi tessellation method, the threedimensional space can be partitioned into the independent Voronoi polyhedra (i.e., the Voronoi cells) centered by each atom [1]. Each Voronoi cell is enveloped by a set of planes bisecting the lines connecting the central atom and its nearest neighboring atoms. The Voronoi polyhedron type $i$ can be identified in terms of the Voronoi index $\left\langle n_{i}{ }^{3}, n_{i}{ }^{4}, n_{i}{ }^{5}, n_{i}{ }^{6}\right\rangle$, where $n_{i}{ }^{k}(k=3,4,5$, 6) denotes the amount of $k$-edged faces in Voronoi polyhedron type $i$. According to the statistical analyses of these Voronoi polyhedra, the preferred polyhedral packing clusters can be regarded as the local geometrical orderings or short-range order of MGs.

Previous simulation results $[35,36]$ also indicate that the five-fold local symmetry parameter is an effective structural parameter, which is highly correlated with the local dynamics and structural relaxation in MG-forming liquids. The local five-fold symmetry parameter is quantified as $f_{i}^{5}=\frac{n_{i}^{5}}{\sum_{k=3,4,5,6} n_{i}{ }^{k}}$ based on the Voronoi tessellation analysis. For a given atomic configuration, if the fraction of the polyhedron type $i$ is symbolized by $P_{i}$, the definition of the average degree of the five-fold local symmetry parameter $W$ can be expressed as

$W=\sum_{i} f_{i}^{5} \times P_{i}$.

Here we followed the previous studies and systematically extracted the system size influences of the local geometrical information at $300 \mathrm{~K}$ based on the related instantaneous atomic configurations of samples with different system sizes.

We first calculated the fractions of the Voronoi polyhedra and differentiated them by the centered-atoms ( $\mathrm{Zr}$ or $\mathrm{Cu}$ ). The fraction histogram of the top ten Voronoi polyhedra in $\mathrm{N} 250$ and $\mathrm{N} 6400$ system at $T=300 \mathrm{~K}$ is shown in Fig. 2a. The populations of the given coordination polyhedra in N250 are almost equal to their counterparts in $\mathrm{N} 6400$, either for $\mathrm{Cu}$-centered polyhedra or the $\mathrm{Zr}$-centered one.

The favored $\mathrm{Cu}$-centered Voronoi clusters are icosahedral or icosahedral-like with a high value of the local five-fold symmetry parameter $f^{5}$. It has been shown that the connectivity of $\mathrm{Cu}$-centered icosahedra exerts a critical influence on the relaxation dynamics and thermal stability $[9,37,38]$. Moreover, Hu et al. [35] proposed that $f^{5} \geq 0.6$ can be a rational and effective threshold for selecting the atoms and illustrated the growing spatial structure correlation during the quenching process. Thus, by choosing the threshold value as $f^{5}=0.6$, we divided the favored $\mathrm{Cu}$-centered Voronoi polyhedra into two categories, and the top ten $\mathrm{Cu}$-centered Voronoi polyhedra in systems N250 and N6400 at $T=300 \mathrm{~K}$ are indicated in Fig. 2b. The negligible difference of the percentage of the $\mathrm{Cu}$-centered Voronoi clusters between two systems indicates that the local structure of the si-
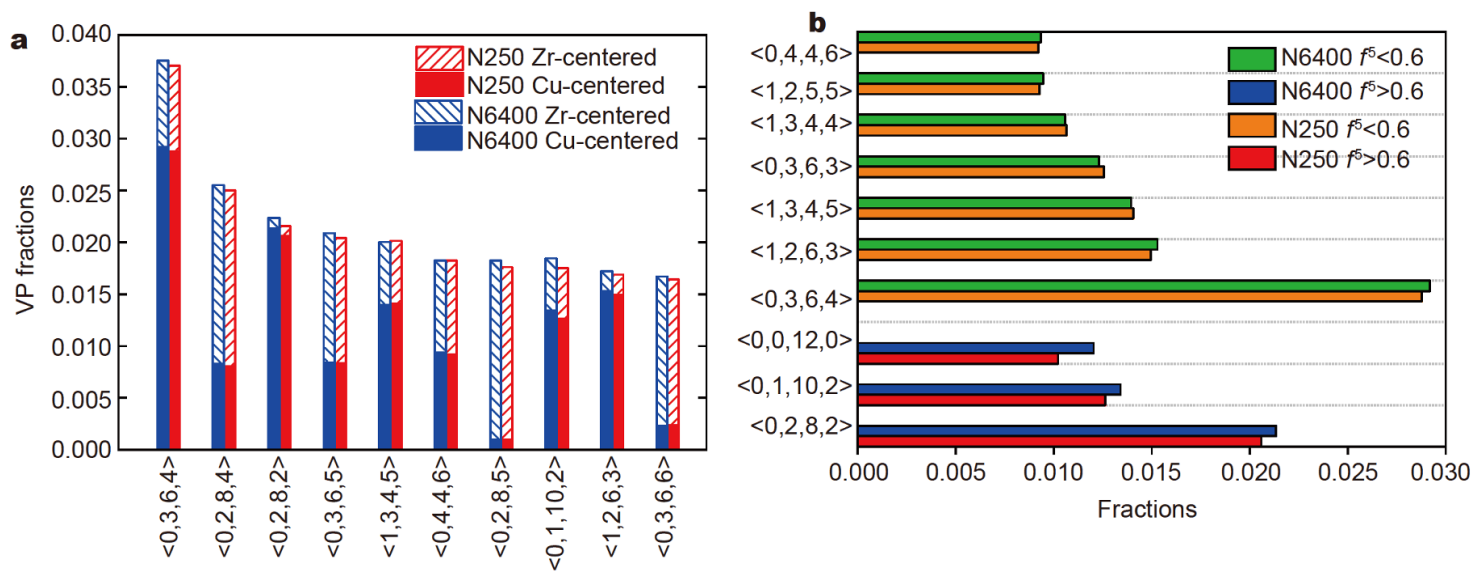

Figure 2 The fraction histogram of the representative Voronoi polyhedra (VP) of systems N250 and N6400 at $T=300 \mathrm{~K}$. (a) The Voronoi polyhedron with the top ten fractions, which is also classified into the $\mathrm{Cu}$-centered clusters and the $\mathrm{Zr}$-centered ones; (b) the top ten $\mathrm{Cu}$-centered Voronoi polyhedra, which are also labeled by the criterion $f^{5} \geq 0.6$ or $f^{5}<0.6$. 


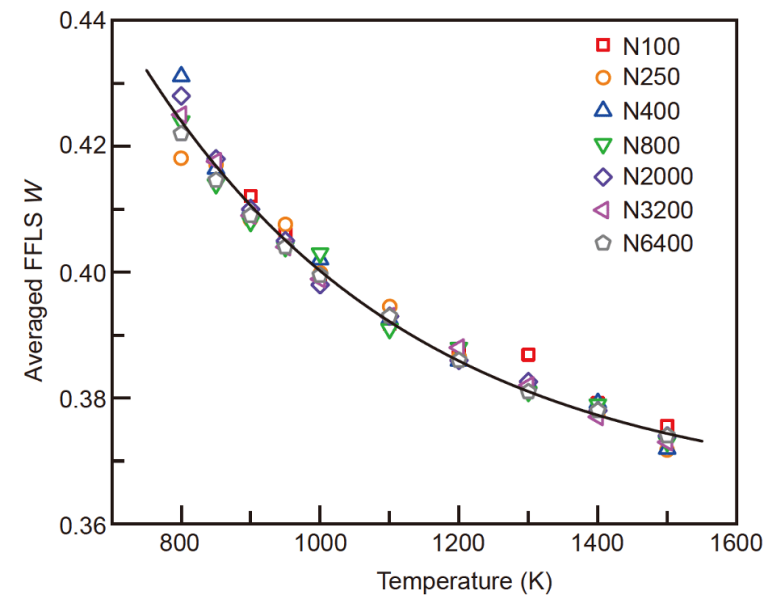

Figure 3 The temperature dependence of structural parameter $W$ characterizing the average degree of the five-fold local symmetry for the simulated systems with different system sizes. The black solid line is a guide for eyes.

mulated $\mathrm{Cu}_{50} \mathrm{Zr}_{50} \mathrm{MG}$ is system-size independent. It was also supported by the average degree of the local five-fold symmetry parameter $W$. As shown in Fig. 3, the temperature dependence of the average degree of the five-fold local symmetry in varying sized systems can collapse into a master curve. The values of structural parameter $W$ at different equilibrium temperatures are roughly close to the earlier study [35]. All these results indicate that the system size has only a negligible effect on the short-range features of atomic structure and the averaged structure features in simulated MGs which is consistent with previous studies [27]. It demonstrates that the AIMD could be an effective method for investigating the local structure information of MGs.

\section{Dynamics}

To explore the finite size effects on the relaxation dynamics, we calculated the mean-squared displacements and the self-diffusion coefficients for all temperatures investigated. The mean-squared displacement (MSD) is defined as:

$\left\langle r^{2}(t)\right\rangle=\frac{1}{N}\left\langle\sum_{i=1}^{N}\left|r_{l}(t)-r_{l}(0)\right|^{2}\right\rangle$,

where $r_{l}(t)$ is the displacement of the particle $l$ during time $t$ and $N$ is the total number of particles. The angle brackets $\langle\cdot\rangle$ denotes the ensemble average over different configurations.

Fig. 4a shows the $\left\langle r^{2}(t)\right\rangle$ against time in a logarithmic scale for three represented systems at 1500 and $800 \mathrm{~K}$. The $\left\langle r^{2}(t)\right\rangle$ curves show a characteristic crossover [39] from the short-time ballistic behavior to the long-time diffusive motion at a high temperature $T=1500 \mathrm{~K}$. There is a prolonged plateau in the intermediate timescale at $800 \mathrm{~K}$, which is relevant to the cage effect [40]. The superposition within the short-time range confirms the negligible system size influence on the local atomic packing behaviors as aforementioned. The deviation of the long-time diffusive process implies the noticeable system size effects of the dynamical properties, especially in supercooled liquids.

The self-diffusion coefficient $D(T)$ is determined directly from the MSD at the long-time region, where the MSD is against time linearly, that is $\left\langle r^{2}(t)\right\rangle \sim t$, also known as the Einstein relation $[41,42]$

$D=\lim _{t \rightarrow \infty} \frac{1}{2 d t}\left\langle\left|r_{i}(t)-r_{i}(0)\right|^{2}\right\rangle$,

where the parameter $d$ denotes the dimensionality. Here, $d=3$ for our systems.

Fig. 4b illustrates the scaled diffusion coefficients $D(N$, $T)$ by the values of the system N6400 at the same temperature $D(N=6400, T)$ versus the system size from 1500 to $800 \mathrm{~K}$. Obviously, for all temperatures investigated, the atomic motion becomes slower as the system size decreases. It suggests that the system size effects cannot be neglected for studying the relaxation dynamics of MGforming liquids.

We further analyzed the system-size dependence of the structural relaxation time $\tau_{\alpha}$ by measuring the self-intermediate scattering function (SISF) for different sized systems. The SISF [31] unveils the density fluctuations of an identical particle in the system over a given time interval $t$. The definition of SISF can be expressed as follows:

$F_{\mathrm{s}}(q, t)=\frac{1}{N}\left\langle\sum_{i=1}^{N} \exp \left[-\mathrm{i} \boldsymbol{q} \cdot \Delta \boldsymbol{r}_{i}(t)\right]\right\rangle$,

where $q$ is the wave vector, $N$ is the number of atoms and $\Delta \boldsymbol{r}_{i}(t)=\boldsymbol{r}_{i}(t)-\boldsymbol{r}_{i}(0)$ represents the displacement vector of atom $i$ in a time interval of $t,\langle\cdot\rangle$ denotes the ensemble average over different configurations. The structural relaxation time $\tau_{\alpha}$ can be derived from the self-intermediate scattering function requiring that the $F_{s}(q, t)$ decays to the $\mathrm{e}^{-1}[40]$. The wave vector $\boldsymbol{q}$ is fixed at $|\boldsymbol{q}|=q_{\max }$, corresponding to the first peak position of the static structure factor. For $\mathrm{Cu}_{50} \mathrm{Zr}_{50}$ system, the calculated $|\boldsymbol{q}|$ is about 2.68 .

Fig. 5a shows the evolution of $F_{s}(q, t)$ with time for three represented systems N250, N2000, and N6400 at T $=1500$ and $800 \mathrm{~K}$. The N250 system represents the small size system, and the N2000 and N6400 systems exemplify 
the medium and the large ones, respectively. At the high temperature $(T=1500 \mathrm{~K})$, the $F_{s}(q, t)$ decays from one to zero exponentially. These curves collapse for three systems which implies the miniscule system-size influence of the relaxation dynamics in high temperature liquids. As the temperature decreases to $T=800 \mathrm{~K}$, the $F_{s}(q, t)$ profiles present typical two-step decay [43] distinguished by the short-time process and the $\alpha$-relaxation at longtime regime. Since the short-time process is correlated with the nearest-neighbor cage effect, the collapse of $F_{\mathrm{s}}(q$, $t$ ) at short-time region implies that the local structural packing is insensitive to the system size, which is consistent with the results of the local structure analysis. However, the deviation of $F_{s}(q, t)$ at the long-time regime indicates the system-size-dependent structural relaxation time in supercooled liquids. The extracted structural relaxation times $\tau_{\alpha}(T, N)$ over the entire range of temperatures are illustrated in Fig. 5b. The data of the sample N100 are absent when the temperature drops below $900 \mathrm{~K}$ due to the crystallization issue. The $\tau_{\alpha}(T, N)$ at high temperature has minimal change with the decreasing system size $N$, but gradually transforms into a more pronounced size dependence as the temperature decreases.

To quantitively characterize the system-size influence, we selected the data of the largest sample N6400 which is closer to the bulk sample as the reference. Fig. $5 \mathrm{c}$ shows the scaled $\alpha$ relaxation times $\tau_{\alpha}(T, N) / \tau_{\alpha}(T, N=6400)$ as a function of the system size from 800 to $1500 \mathrm{~K}$. Here, the scaling parameter $\gamma=\tau_{\alpha}(T, N) / \tau_{\alpha}(T, N=6400)$ can be regarded as the degree of deviation from the bulk value. The scaled values $\tau_{\alpha}(T, N) / \tau_{\alpha}(T, N=6400)$ start to be greater than 1 as the system size $N$ reduces to 1000 atoms. The value increases rapidly as the system size decreases, which indicates the slower dynamics in smaller systems. It signifies the pronounced finite size effects of relaxation dynamics which is consistent with the results in Fig. $4 \mathrm{~b}$. Moreover, we further plotted the parameter $\gamma$ as a function of the equilibrium temperature for systems N400, N800, N2000, and N3200. The results are shown in Fig. $5 \mathrm{~d}$. The deviation of the structural relaxation times from the bulk value extends to a higher temperature with decreasing system size $N$. In previous studies [21,23], the characteristic correlation length of dynamics in supercooled liquid grows rapidly during the quenching process. As the growing correlation length becomes comparable to the box size, the system size effect dominates the relaxation dynamics as shown in Fig. 5. It means that the finite size effects of the dynamical properties in a simulated box containing hundreds of atoms with periodic boundary conditions cannot be neglected, especially in supercooled liquids. For representing the dynamic behaviors in the bulk MG systems, the model system is supposed to contain at least thousands of atoms to avoid the boundary effects.

\section{Structure-dynamics relation}

Recent studies [2,36-38] have demonstrated that shortrange order is inadequate to give a reasonable interpretation for the relaxation dynamics in MGs. The dynamical slowdown approach to the glass transition in supercooled liquids is determined by the static configuration correlation length rather than the local structural orderings [28]. Wei et al. [44] ascertained the weak correlation between short-range orders and the atomic dynamic process in terms of the wide-distributed activation energy spectrum of one specific atom in MG. Since we had investigated the system size dependence of short-
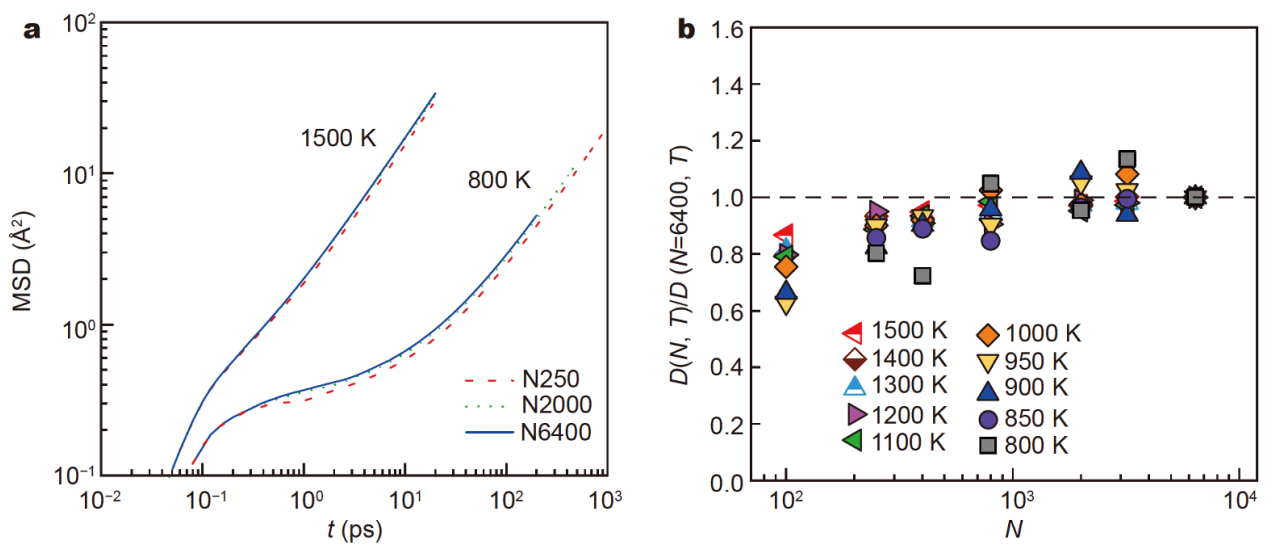

Figure 4 System size dependence of (a) the atomic mobility in terms of MSD and (b) the scaled self-diffusion coefficients for all temperatures investigated. 

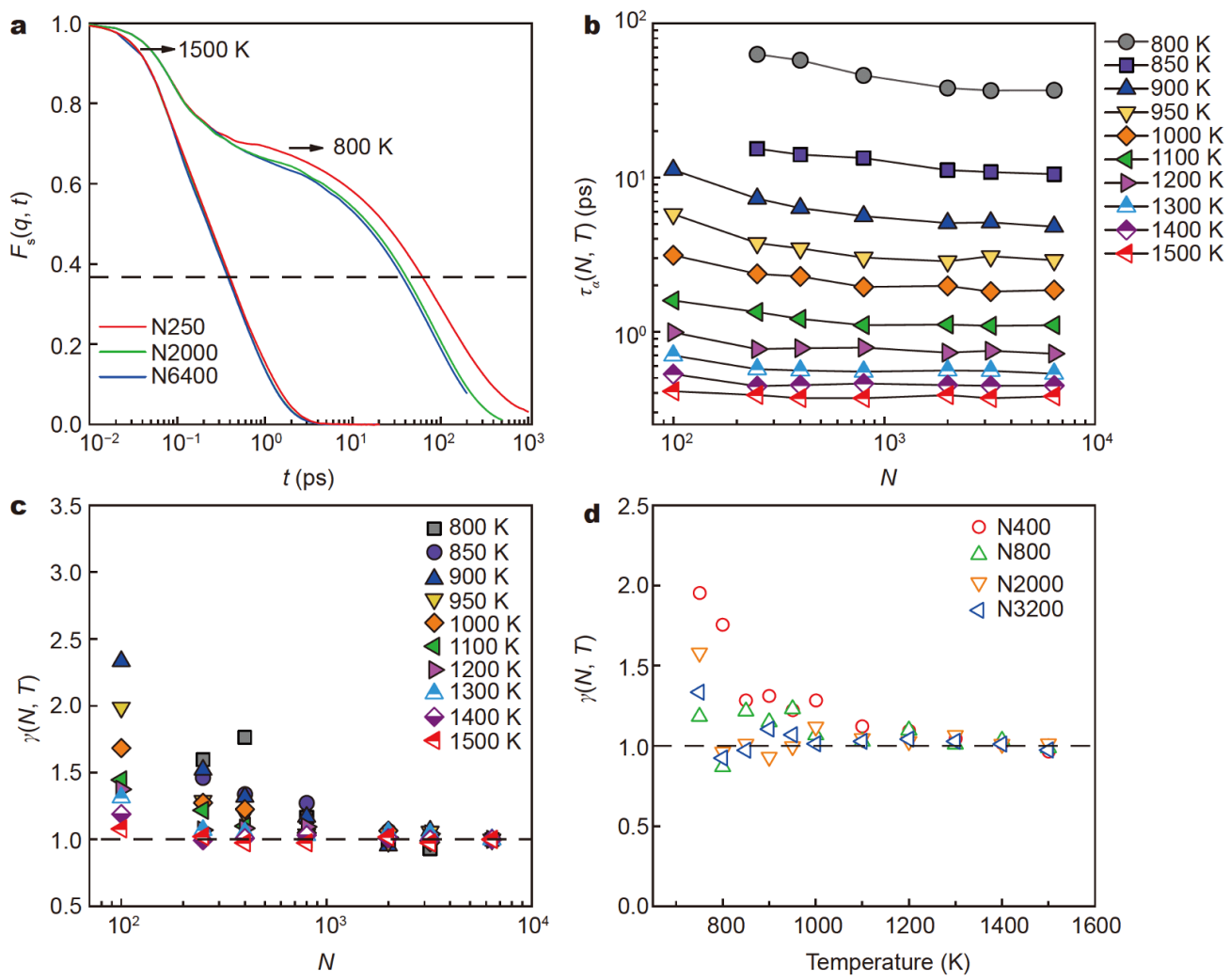

Figure 5 System size dependence of the relaxation dynamics of bulk $\mathrm{Cu}_{50} \mathrm{Zr}_{50}$ systems. (a) The self-intermediate scattering function $F_{s}(q, t)$ for three $\mathrm{Cu}_{50} \mathrm{Zr}_{50}$ MG-forming liquids at $T=1500 \mathrm{~K}$ and $T=800 \mathrm{~K}$. The selected systems are N250, N2000, and N6400. The structural relaxation time is determined as the $F_{\mathrm{s}}(q, t)$ decays to $\mathrm{e}^{-1}$ (marked with the horizontal dashed line). (b) The system-size dependence of structural relaxation times $\tau_{\alpha}(T$, $N$ ) from the high-temperature liquid $T=1500 \mathrm{~K}$, down to the near- $T_{\mathrm{g}}$ temperature $T=800 \mathrm{~K}$. (c) The plots of scaled $\alpha$-relaxation times versus the system size $N$ at various temperatures. (d) The temperature evolution of the scaled structural relaxation times $\tau_{\alpha}(T, N) / \tau_{\alpha}(T, N=6400)$ for four represented systems (N400, N800, N2000, and N3200).

range order and the relaxation dynamics simultaneously, it is intriguing to further examine the system size effects of the relation between the local structure and the structural relaxation.

As mentioned above, the static structural properties, such as the local geometrical orderings, are independent of the system size.

However, as shown in Fig. 6, the scaling parameter $\gamma=$ $\tau_{\alpha}(T, N) / \tau_{\alpha}(T, N=6400)$, which characterizes the system size effects of relaxation dynamics, indicates the combined influences of the system size and temperature. It suggests that the relations between the local structure and structural dynamics are discrepant in systems with various sizes, which manifests the decoupling between the local structural orderings and dynamics in MGs. Moreover, as the temperature decreases, the system size for eliminating the boundary effects of dynamical properties increases. The number of atoms in a cubic simulated model should be over thousands for investigating the structural origins of dynamics in MGs. It challenges the application of atomistic numerical simulations in multicomponent MGs.

\section{Correlation between structural relaxation time and dynamic heterogeneity}

Heterogeneity, an intrinsic nature for amorphous materials, establishes a general connection between spatially heterogeneous dynamics and nonexponential relaxation $[43,45]$. It had been comprehensively analyzed in supercooled liquids via experimental techniques and computational simulations [46-49]. Wang et al. [50] reported a general correlation between the dynamic heterogeneity and relaxation time in a variety of model glass-forming liquids with pairwise potentials by using the identical dynamic heterogeneity condition, which was also confirmed in MG-forming systems [51] with many-body potentials. However, how the system size affects this correlation in the MG system is still unclear. To address 


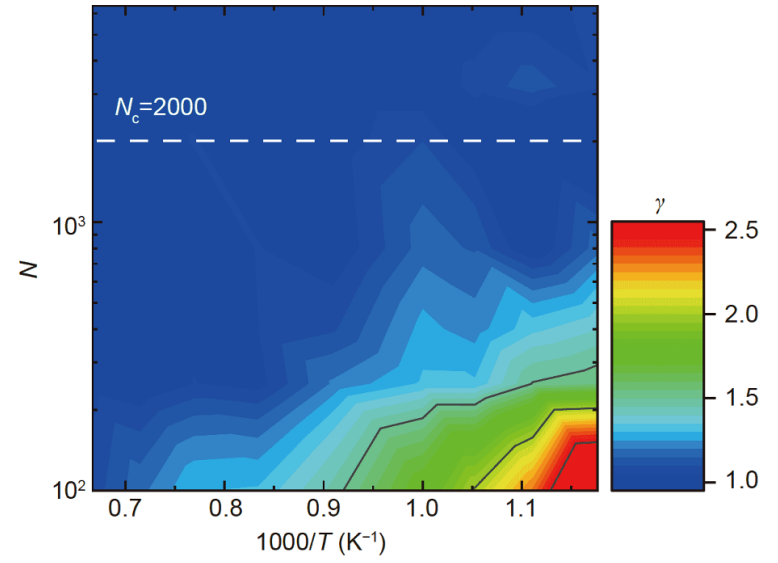

Figure 6 The two-dimensional contour map of the structural relaxation times as the functions of system size $N$ and scaled reciprocal temperature $1000 / T$ in a semi-logarithmic scale. The parameter $\gamma=\tau_{\alpha}(T$, $N) / \tau_{\alpha}(T, N=6400)$ and the horizontal dotted line is a guide for $N_{\mathrm{c}}=$ 2000 .

this issue, we introduced the non-Gaussian parameter to unravel the dynamic heterogeneity.

The significant deviations from Gaussian approxima- tion of the self-part of the van Hove correlation function suggest the existence of nontrivial spatiotemporal fluctuations in glass-forming liquids [49,52]. The degree of deviations can be characterized by the time-dependent non-Gaussian parameter $[53,54] \quad \alpha_{2}(t)=\frac{3\left\langle r^{4}(t)\right\rangle}{5\left\langle r^{2}(t)\right\rangle^{2}}-1$, where $r(t)$ is the displacement of a particle during time $t$.

Fig. $7 \mathrm{a}$ and $\mathrm{b}$ show the time dependence of the nonGaussian parameter $\alpha_{2}$ for three represented systems (N250, N2000, and N6400) at 1500 and $800 \mathrm{~K}$, respectively. With the temperature decreasing, the peak value of the non-Gaussian parameter $\alpha_{2, \max }$ increases, accompanied by the characteristic time $t^{*}$ corresponding to the peak value extending to a longer time scale. It indicates that the dynamic heterogeneity tends to get stronger as the temperature decreases. We can conjecture that, from the time evolution of non-Gaussian parameter for three chosen systems at identical temperature, the $\alpha_{2, \max }$ increases with the system size decreasing. The system size dependence of the $\alpha_{2, \max }$ at different temperatures is
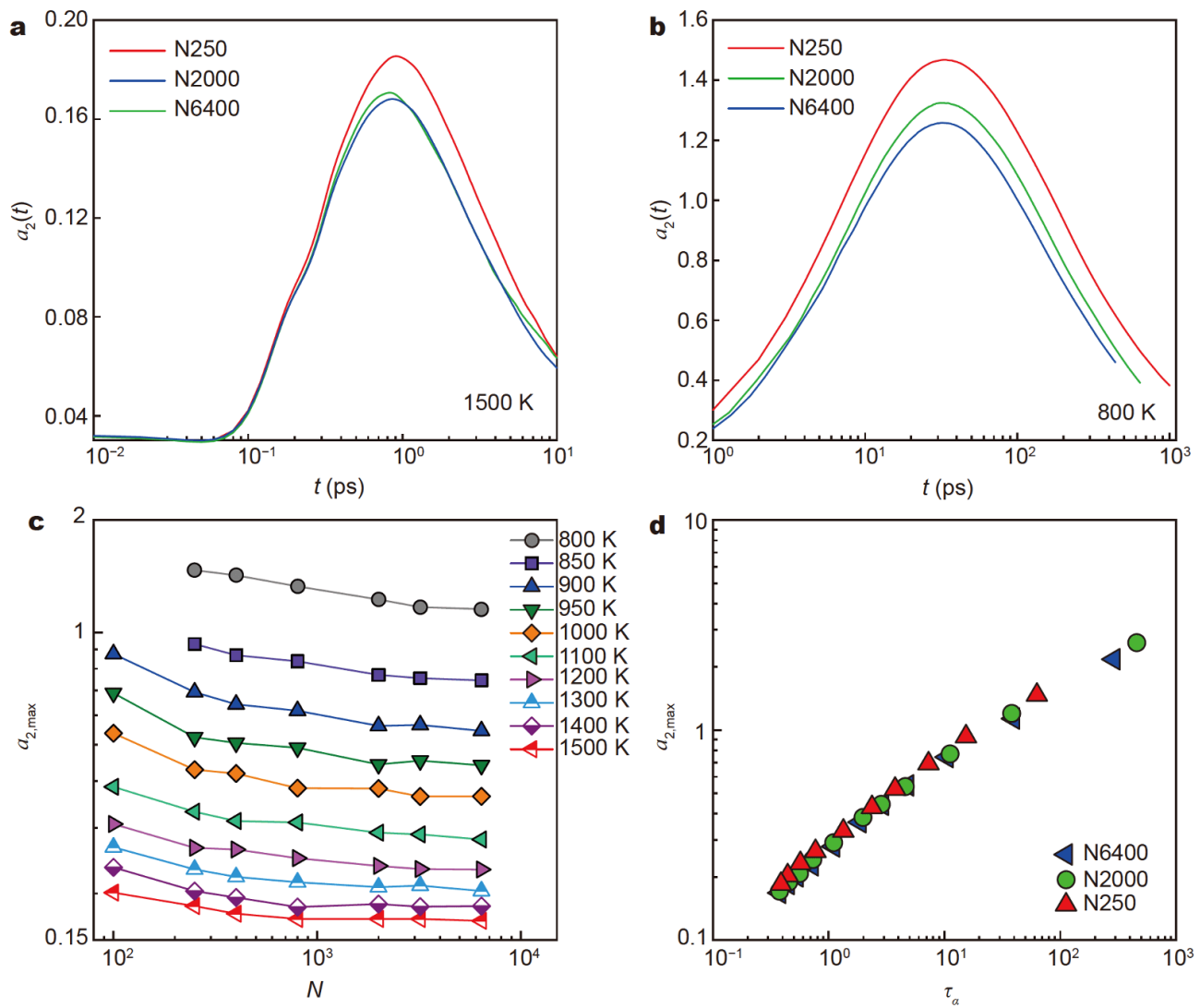

Figure 7 The correlation between structural relaxation time and dynamic heterogeneity. The extracted non-Gaussian parameter $\alpha_{2}$ against time $t$ at (a) $T=1500 \mathrm{~K}$ and (b) $T=800 \mathrm{~K}$. (c) The system size dependence of the maximum of non-Gaussian parameter $\alpha_{2, \max }$ for all equilibrium temperatures investigated. (d) Log-log plot of $\alpha_{2, \max }$ versus relaxation time $\tau_{\alpha}$. 
shown in Fig. 7c. The measured $\alpha_{2, \max }$ shows a descending tendency until a plateau is reached at a crossover system size. The crossover system size is enlarged along with the equilibrium temperature decreasing.

Fig. $7 \mathrm{~d}$ shows the maximum of non-Gaussian parameter $\alpha_{2, \max }$ increases monotonously with the structural relaxation times $\tau_{\alpha}$, which is reminiscent of the correlation between dynamic heterogeneity and dynamics slowdown $[50,55]$. Moreover, the existence of a general link between $\alpha_{2, \max }$ and $\tau_{\alpha}$ can be perceived in terms of the collapse of curves for three systems with different sizes. It indicates the generalization of the link between the structural relaxation time and dynamic heterogeneity in MG-forming liquids.

\section{CONCLUSIONS}

In summary, we presented a molecular-dynamics study to systematically explore the finite size effects of the structural and dynamical properties in a model $\mathrm{Cu}_{50} \mathrm{Zr}_{50}$ bulk MG system. Our results demonstrated that the structural indicators of short-range orders are insensitive to the change of system size. Moreover, the system-size effects are extensive in the dynamical phenomena, including atomic diffusion, structural relaxation, and dynamic heterogeneity. The correlation between the relaxation dynamics and dynamic heterogeneity is qualitatively indicated, which verifies the generalization of the link between the structural relaxation time and dynamic heterogeneity in MG-forming liquids as recently proposed in Ref. [51].

We further uncover the decoupling between static structure and relaxation dynamics in the context of the scarce system size. It can be anticipated that the structural mechanism in relaxation dynamics originated from highorder correlations and medium-range orders. To characterize the structural origins of dynamics in bulk MGs, the system size should consist of thousands of atoms, which is far beyond the limits of system size in AIMD. The structural information in the medium-range order scale may better reveal the dynamics behavior in metallic liquids and glasses. Former studies demonstrated that the local environments of atomic clusters have a different impact on dynamics in MG-forming liquids [9]. The adoption of local connectivity based on graph theory $[9,11,38]$ provides a novel perspective to understand this long-standing subject which remains to further investigation.

Concerning the exploration of structure-dynamics relationship in molecular simulations, hitherto, another conundrum is the multi-component interatomic poten- tials fail to accurately describe the physical properties in full composition range. The neural-networks potential and other machine learning-based force fields are expected to be the powerful icebreaker.

\section{Received 4 September 2020; accepted 10 October 2020;} published online 5 January 2021

1 Cheng YQ, Ma E. Atomic-level structure and structure-property relationship in metallic glasses. Prog Mater Sci, 2011, 56: 379-473

2 Ding J, Ma E. Computational modeling sheds light on structural evolution in metallic glasses and supercooled liquids. npj Comput Mater, 2017, 3: 9

3 Guan PF, Fujita T, Hirata A, et al. Structural origins of the excellent glass forming ability of $\mathrm{Pd}_{40} \mathrm{Ni}_{40} \mathrm{P}_{20}$. Phys Rev Lett, 2012, 108: 175501

4 Tanaka H, Tong H, Shi R, et al. Revealing key structural features hidden in liquids and glasses. Nat Rev Phys, 2019, 1: 333-348

5 Allen MP, Tildesley DJ. Computer Simulation of Liquids. Oxford: Oxford University Press, 1989

6 Mendelev MI, Han S, Srolovitz DJ, et al. Development of new interatomic potentials appropriate for crystalline and liquid iron. Philos Mag, 2003, 83: 3977-3994

7 Mendelev MI, Kramer MJ, Ott RT, et al. Development of suitable interatomic potentials for simulation of liquid and amorphous $\mathrm{Cu}$ Zr alloys. Philos Mag, 2009, 89: 967-987

8 Jakse N, Pasturel A. Local order and dynamic properties of liquid and undercooled $\mathrm{Cu}_{x} \mathrm{Zr}_{1-x}$ alloys by $a b$ initio molecular dynamics. Phys Rev B, 2008, 78: 214204

9 Li FX, Li MZ. Local environments of atomic clusters and the effect on dynamics in CuZr metallic glass-forming liquids. J Appl Phys, 2017, 122: 225103

10 Peng HL, Li MZ, Wang WH. Structural signature of plastic deformation in metallic glasses. Phys Rev Lett, 2011, 106: 135503

$11 \mathrm{Wu} \mathrm{ZW}, \mathrm{Kob} \mathrm{W}$, Wang WH, et al. Stretched and compressed exponentials in the relaxation dynamics of a metallic glass-forming melt. Nat Commun, 2018, 9: 5334

$12 \mathrm{Wu}$ ZW, Li R. Revisiting the breakdown of Stokes-Einstein relation in glass-forming liquids with machine learning. Sci China-Phys Mech Astron, 2020, 63: 276111

13 Jakse N, Pasturel A. Local order of liquid and supercooled zirconium by ab initio molecular dynamics. Phys Rev Lett, 2003, 91: 195501

14 Li M, Wang CZ, Hao SG, et al. Structural heterogeneity and medium-range order in $\mathrm{Zr}_{x} \mathrm{Cu}_{100-x}$ metallic glasses. Phys Rev B, 2009, 80: 184201

15 Ganesh P, Widom M. Ab initio simulations of geometrical frustration in supercooled liquid $\mathrm{Fe}$ and Fe-based metallic glass. Phys Rev B, 2008, 77: 014205

16 Jakse N, Le Bacq O, Pasturel A. Prediction of the local structure of liquid and supercooled tantalum. Phys Rev B, 2004, 70: 174203

17 Cheng YQ, Ma E, Sheng HW. Atomic level structure in multicomponent bulk metallic glass. Phys Rev Lett, 2009, 102: 245501

18 Kresse G. Ab initio molecular dynamics: Recent progresses and limitations. J Non-Crystalline Solids, 2002, 312-314: 52-59

19 Román FL, White JA, González A, et al. Fluctuations in a small hard-disk system: Implicit finite size effects. J Chem Phys, 1999, 110: 9821-9824

20 Asta AJ, Levesque M, Vuilleumier $\mathrm{R}$, et al. Transient hydro- 
dynamic finite-size effects in simulations under periodic boundary conditions. Phys Rev E, 2017, 95: 061301

21 Berthier L, Biroli G, Coslovich D, et al. Finite-size effects in the dynamics of glass-forming liquids. Phys Rev E, 2012, 86: 031502

22 Horbach J, Kob W, Binder K, et al. Finite size effects in simulations of glass dynamics. Phys Rev E, 1996, 54: R5897-R5900

23 Kim K, Yamamoto R. Apparent finite-size effects in the dynamics of supercooled liquids. Phys Rev E, 2000, 61: R41-R44

24 Taamalli S, Hinds J, Migirditch S, et al. Enhanced diffusion in finite-size simulations of a fragile diatomic glass former. Phys Rev E, 2016, 94: 052604

25 Li YZ, Sun YT, Lu Z, et al. Size effect on dynamics and glass transition in metallic liquids and glasses. J Chem Phys, 2017, 146: 224502

26 Zhang WB, Liu J, Lu SH, et al. Size effect on atomic structure in low-dimensional Cu-Zr amorphous systems. Sci Rep, 2017, 7: 7291

27 Amokrane S, Ayadim A, Levrel L. Structure of the glass-forming metallic liquids by ab-initio and classical molecular dynamics, a case study: Quenching the $\mathrm{Cu}_{60} \mathrm{Ti}_{20} \mathrm{Zr}_{20}$ alloy. J Appl Phys, 2015, 118: 194903

$28 \mathrm{Hu}$ YC, Li YW, Yang Y, et al. Configuration correlation governs slow dynamics of supercooled metallic liquids. Proc Natl Acad Sci USA, 2018, 115: 6375-6380

29 Plimpton S. Fast parallel algorithms for short-range molecular dynamics. J Comput Phys, 1995, 117: 1-19

30 Hoover WG. Canonical dynamics: Equilibrium phase-space distributions. Phys Rev A, 1985, 31: 1695-1697

31 Jaiswal A, Egami T, Zhang Y. Atomic-scale dynamics of a model glass-forming metallic liquid: Dynamical crossover, dynamical decoupling, and dynamical clustering. Phys Rev B, 2015, 91: 134204

32 Lou $\mathrm{H}$, Wang $\mathrm{X}$, Cao Q, et al. Negative expansions of interatomic distances in metallic melts. Proc Natl Acad Sci USA, 2013, 110: 10068-10072

33 Pan SP, Qin JY, Wang WM, et al. Origin of splitting of the second peak in the pair-distribution function for metallic glasses. Phys Rev B, 2011, 84: 092201

34 Li MZ, Peng HL, Hu YC, et al. Five-fold local symmetry in metallic liquids and glasses. Chin Phys B, 2017, 26: 016104

35 Hu YC, Li FX, Li MZ, et al. Five-fold symmetry as indicator of dynamic arrest in metallic glass-forming liquids. Nat Commun, 2015, 6: 8310

$36 \mathrm{Hu}$ YC, Li FX, Li MZ, et al. Structural signatures evidenced in dynamic crossover phenomena in metallic glass-forming liquids. J Appl Phys, 2016, 119: 205108

37 Soklaski R, Nussinov Z, Markow Z, et al. Connectivity of icosahedral network and a dramatically growing static length scale in Cu-Zr binary metallic glasses. Phys Rev B, 2013, 87: 184203

$38 \mathrm{Wu} \mathrm{ZW}$, Li MZ, Wang WH, et al. Correlation between structural relaxation and connectivity of icosahedral clusters in CuZr metallic glass-forming liquids. Phys Rev B, 2013, 88: 054202

39 Del Gado E, Kob W. Length-scale-dependent relaxation in colloidal gels. Phys Rev Lett, 2007, 98: 028303

40 Horbach J, Kob W. Relaxation dynamics of a viscous silica melt: The intermediate scattering functions. Phys Rev E, 2001, 64: 041503

41 Lee HS, Tuckerman ME. Dynamical properties of liquid water from $a b$ initio molecular dynamics performed in the complete basis set limit. J Chem Phys, 2007, 126: 164501

42 Mendelev MI, Kramer MJ, Ott RT, et al. Molecular dynamics si- mulation of diffusion in supercooled $\mathrm{Cu}-\mathrm{Zr}$ alloys. Philos Mag, 2009, 89: 109-126

43 Royall CP, Williams SR. The role of local structure in dynamical arrest. Phys Rep, 2015, 560: 1-75

44 Wei D, Yang J, Jiang MQ, et al. Revisiting the structure-property relationship of metallic glasses: Common spatial correlation revealed as a hidden rule. Phys Rev B, 2019, 99: 014115

45 Debenedetti PG, Stillinger FH. Supercooled liquids and the glass transition. Nature, 2001, 410: 259-267

46 Berthier L, Biroli G. Theoretical perspective on the glass transition and amorphous materials. Rev Mod Phys, 2011, 83: 587-645

47 Schmidt-Rohr K, Spiess HW. Nature of nonexponential loss of correlation above the glass transition investigated by multidimensional NMR. Phys Rev Lett, 1991, 66: 3020-3023

48 Tracht U, Wilhelm M, Heuer A, et al. Length scale of dynamic heterogeneities at the glass transition determined by multidimensional nuclear magnetic resonance. Phys Rev Lett, 1998, 81: 2727-2730

49 Chaudhuri P, Berthier L, Kob W. Universal nature of particle displacements close to glass and jamming transitions. Phys Rev Lett, 2007, 99: 060604

50 Wang $\mathrm{L}, \mathrm{Xu} \mathrm{N}$, Wang WH, et al. Revealing the link between structural relaxation and dynamic heterogeneity in glass-forming liquids. Phys Rev Lett, 2018, 120: 125502

51 Ren N, Hu L, Wang L, et al. Revealing a hidden dynamic signature of the non-Arrhenius crossover in metallic glass-forming liquids. Scripta Mater, 2020, 181: 43-47

52 Kob W, Donati C, Plimpton SJ, et al. Dynamical heterogeneities in a supercooled Lennard-Jones liquid. Phys Rev Lett, 1997, 79: 28272830

53 Donati C, Glotzer SC, Poole PH, et al. Spatial correlations of mobility and immobility in a glass-forming Lennard-Jones liquid. Phys Rev E, 1999, 60: 3107-3119

54 Rahman A. Correlations in the motion of atoms in liquid argon. Phys Rev, 1964, 136: A405-A411

55 Flenner E, Szamel G. Dynamic heterogeneity in a glass forming fluid: Susceptibility, structure factor, and correlation length. Phys Rev Lett, 2010, 105: 217801

Acknowledgements This work was supported by the Science Challenge Project (TZ2018004) and the NSAF joint Program (U1930402). We also acknowledge the computational support from Beijing Computational Research Center (CSRC). Insightful discussions with members of the "Beijing Metallic Glass Club" (Wei-hua Wang, Lijin Wang, Shan Zhang, Nannan Ren, Xuerui Wei, and Qi Liu) are highly acknowledged.

Author contributions Guan P designed and supervised the work. Sun $\mathrm{S}$ conducted the calculations and analyzed the data. Sun S and Guan P wrote the paper.

Conflict of interest The authors declare that they have no conflict of interest. 


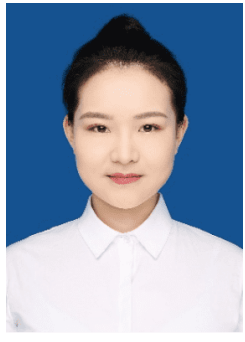

Shengjun Sun is currently a $\mathrm{PhD}$ candidate at the Materials and Energy Division, Beijing Computational Science Research Center, Beijing, China. She received her bachelor degree from Beijing University of Technology, Beijing, China in 2017. Her $\mathrm{PhD}$ research focuses on the intrinsic heterogeneity of the metallic glasses and the metallic glass-forming liquids.

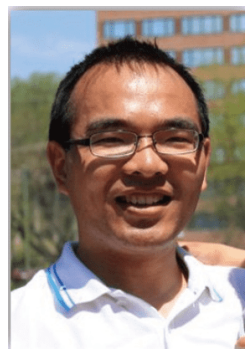

Pengfei Guan obtained his bachelor and master degree from Jilin University, Changchun, China and $\mathrm{PhD}$ from the Central Iron \& Steel Research Institute, Beijing, China. He is currently a full professor at Beijing Computational Science Research Center, Beijing, China. His research focuses on computational materials science towards the theoretical design of high-performance alloys, such as metallic glasses and highstrength alloys.
块体金属玻璃结构-动力学关联模拟研究的临界 模型尺度

孙胜君, 管鹏飞*

摘要 原子尺度模拟是当前理解金属玻璃体系的无序结构及其构 效关系的有效方法. 基于分子动力学模拟, 本文以代表性金属玻璃 $\mathrm{Cu}_{50} \mathrm{Zr}_{50}$ 为研究对象, 系统地研究了块体金属玻璃体系的原子结构 和动力学行为的有限尺度效应. 通过研究发现, 其结构短程序对模 型尺寸并不敏感, 但其动力学性质却表现出明显的尺寸相关性. 通 过定量计算可知，当立方体模型所含原子数低于 2000时，金属玻 璃体系的结构短程序特征和结构弛豫之间的相关性发生退简并, 但其结构弛豫时间和动力学非均匀性依然遵循简并的普适关系. 本文的结论给出了结构弛豫和动力学非均匀性之间存在本征关联 的有力证据，并第一次明确给出了在没有表面效应的情况下基于 计算模拟研究块体金属玻璃结构、动力学及其关联所需要的临界 模型尺度. 